\title{
Intermolecular potential parameters and combining rules determined from viscosity data
}

\author{
Lucas A. J. Bastien, Phillip N. Price, Nancy J. Brown* \\ Atmospheric Sciences Department \\ Environmental Energy Technology Division \\ Lawrence Berkeley National Laboratory \\ Berkeley California, 94707 \\ lucas.bastien@insa-lyon.fr
}

PNPrice@lbl.gov

NJBrown@1bl.gov

Revised Version: May 27, 2010

Accepted for publication in the International Journal of Chemical Kinetics

* Corresponding author: Nancy J. Brown; MS 90 K-110, Berkeley California 94720-8108;

NJBrown@lbl.gov; 510-486-4241 Fax: 510-486-5928 


\section{Abstract}

The Law of Corresponding States has been demonstrated for a number of pure substances and binary mixtures, and provides evidence that the transport properties viscosity and diffusion can be determined from a molecular shape function, often taken to be a Lennard-Jones 12-6 potential, that requires two scaling parameters: a well depth $\varepsilon_{\mathrm{ij}}$ and a collision diameter $\sigma_{\mathrm{ij}}$, both of which depend on the interacting species $i$ and $j$. We obtain estimates for $\varepsilon_{\mathrm{ij}}$ and $\sigma_{\mathrm{ij}}$ of interacting species by finding the values that provide the best fit to viscosity data for binary mixtures, and compare these to calculated parameters using several "combining rules" that have been suggested for determining parameter values for binary collisions from parameter values that describe collisions of like molecules. Different combining rules give different values for $\sigma_{\mathrm{ij}}$ and $\varepsilon_{\mathrm{ij}}$ and for some mixtures the differences between these values and the best-fit parameter values are rather large. There is a curve in $\left(\varepsilon_{\mathrm{ij}}, \sigma_{\mathrm{ij}}\right)$ space such that parameter values on the curve generate a calculated viscosity in good agreement with measurements for a pure gas or a binary mixture. The various combining rules produce couples of parameters $\varepsilon_{\mathrm{ij}}, \sigma_{\mathrm{ij}}$ that lie close to the curve and therefore generate predicted mixture viscosities in satisfactory agreement with experiment. Although the combining rules were found to underpredict the viscosity in most of the cases, Kong's rule was found to work better than the others, but none of the combining rules consistently yields parameter values near the best-fit values, suggesting that improved rules could be developed.

Keywords: viscosity, transport properties, combining rules, intermolecular potential parameters 


\section{I - Introduction}

Many important phenomena that occur in gas mixtures depend on molecular transport processes including viscosity, diffusion, and thermal conductivity. Transport properties are often critically important in engineering applications and for understanding phenomena like combustion processes, hypersonic flows, and chemical vapor deposition. A recent trend in engineering design is to use modeling to reduce design times, and combustion is an area benefitting from this approach. Modeling combustion processes requires accurate values of transport properties over a wide range of temperatures and pressures. It is not possible to measure all the requisite transport properties, so we must have models to calculate them. This requires adequate information about the intermolecular potential and the underlying dynamics.

Kinetic theory allows the prediction of transport properties of a mixture, if the potential energy of molecular interactions in the mixture is known as a function of intermolecular separation and orientation. A description of the potential as a function of intermolecular separation $r$, averaged over molecular orientations, suffices to calculate viscosity and diffusion coefficients.

Potentials of the form $V(r)=\varepsilon_{i j} \varphi\left(r / \sigma_{i j}\right)$ were proposed by Brown and Munn [1] to compute transport properties, where $i$ and $j$ indicate the chemical species of the two interacting molecules. The shape function, $\varphi$, is frequently taken to be a Lennard-Jones 12-6 potential, where the energy scaling parameter $\varepsilon_{\mathrm{ij}}$ is the well depth, and $\sigma_{\mathrm{ij}}$ is the length scaling parameter that defines the intermolecular separation at which the potential is zero. Frequently the parameters are obtained by finding the values such that predictions of bulk properties (second virial coefficient and transport properties) provide the best possible fit to experiment. We focus on viscosity data because, according to the excellent review by Wakeham et al. (2007) [2], the errors associated 
with viscosity measurements post 1972 are less than $0.5 \%$, and Kestin et al. (1977) [3], measured the viscosity of eighteen binary mixtures with a claimed uncertainty of only $0.2 \%$.

Mourits and Rummens [4] review intermolecular potentials based upon viscosity measurements, discuss the problem of indeterminacy inherent to the calculations, but do not characterize the nature of the indeterminacy. Indeterminacy in this context implies that many combinations of the potential parameters, $\varepsilon_{\mathrm{i}}$ and $\sigma_{\mathrm{i}}$, can be used to calculate viscosities of acceptable precision (2\% or less) for species i.

The non-uniqueness or indeterminacy of the potential parameters has not been recognized in many disciplines like the combustion [5-8] and chemical physics [9] communities that use transport properties in various applications. Kim and Ross [10] and Barker and Pompe [11] found that diffusion coefficients are remarkably insensitive to the intermolecular potential.

Mourtis and Rumens further indicate the greater difficulty in finding suitable potential parameters for mixtures. Combination rules are often used to infer potential parameters for unlike molecules from those associated with like molecules, and there has long been debate about the suitability of different combination rules $[6,12,13]$.

Viscosity measurements of a single chemical species allow direct estimation of the parameters that describe the interaction between two molecules of the same species, $\varepsilon_{\mathrm{ii}}$ and $\sigma_{\mathrm{ii}}$. The situation is more complicated for even the simplest mixture: since every type of molecule interacts with every other type (and with its own type), a mixture of $N$ species requires $N(N+1) / 2 \varepsilon_{\mathrm{ij}}$ and $\sigma_{\mathrm{ij}}$ values. We first fit $\varepsilon$ and $\sigma$ to viscosity data for both pure species followed by fitting $\varepsilon_{\mathrm{ij}}$ and $\sigma_{\mathrm{ij}}$ to viscosities for binary mixtures, and use the results to investigate the accuracy of "combining 
rules" that determine $\varepsilon_{\mathrm{ij}}$ and $\sigma_{\mathrm{ij}}$ for collisions between unlike molecules from values for like molecules: given $\left(\varepsilon_{\mathrm{ii}}, \sigma_{\mathrm{ii}}\right)$ and $\left(\varepsilon_{\mathrm{jj}}, \sigma_{\mathrm{jj}}\right)$, what is the best estimate of $\left(\varepsilon_{\mathrm{ij}}, \sigma_{\mathrm{ij}}\right)$ ?

Seven non-polar species for which highly accurate viscosity data are available are considered: Ar, $\mathrm{N}_{2}, \mathrm{O}_{2}, \mathrm{CO}_{2}, \mathrm{CH}_{4}, \mathrm{CF}_{4}$, and $\mathrm{SF}_{6}$ and 12 of their binary mixtures: Ar- $\mathrm{CF}_{4}, \mathrm{Ar}_{-} \mathrm{SF}_{6}, \mathrm{CF}_{4}-\mathrm{SF}_{6}$, $\mathrm{CH}_{4}-\mathrm{CF}_{4}, \mathrm{CH}_{4}-\mathrm{SF}_{6}, \mathrm{CO}_{2}-\mathrm{CF}_{4}, \mathrm{CO}_{2}-\mathrm{SF}_{6}, \mathrm{~N}_{2}-\mathrm{CF}_{4}, \mathrm{~N}_{2}-\mathrm{SF}_{6}, \mathrm{O}_{2}-\mathrm{CF}_{4}, \mathrm{O}_{2}-\mathrm{CO}_{2}$, and $\mathrm{O}_{2}-\mathrm{SF}_{6}$. Data sources are listed in Table I. The viscosities are measured at atmospheric pressure, and the temperature range of interest is $1<T^{*}<10$ where $T^{*}$, the reduced temperature, is defined as $T^{*}=k_{B} T / \mathcal{E}$. We obtain estimates for $\varepsilon_{\mathrm{ij}}$ and $\sigma_{\mathrm{ij}}$ for each pair of interacting species by finding the values that provide the best fit to the viscosity data. Our estimates are compared to results given in the literature and to the values calculated using the two widely-used computational approaches for the prediction of transport properties: the TRANLIB package of Kee et al. [14], which is oriented toward combustion modeling; and the semi-empirical approach developed by Najafi et al. [15], Boushehri et al. [16], Bzowski et al. [17], and Mason and Uribe [18], which we refer to as MKC (Mason, Kestin, and Colleagues).

Our contributions include the development of inverse methods for determining $\varepsilon_{\mathrm{ij}}$ and $\sigma_{\mathrm{ij}}$; a physical explanation of the non-uniqueness of $(\varepsilon, \sigma)$ for predicting viscosity at a given temperature; and comparison of empirically determined values of $\left(\varepsilon_{\mathrm{ij}}, \sigma_{\mathrm{ij}}\right)$ to the values predicted by commonly used combining rules. We demonstrate even though different combination rules produce different potential parameters for binary systems, many of these result in acceptable values of the binary transport coefficients. 


\section{II - Theory}

\section{1) Transport properties equations}

The formulae given in this section are appropriate under the assumption that the properties of a mixture can be determined from two-body collisions, a condition that holds for most mixtures except at high pressure, where three-body collisions can be important. For pure species, all collisions are between like molecules so we drop the indices on $\varepsilon_{\mathrm{ii}}$ and $\sigma_{\mathrm{ii}}$. The viscosity $\eta$ of a pure gas at temperature $T$, is given by the kinetic theory of gases as the second order Kihara solution as:

$$
\eta(T)=\frac{5}{16} \sqrt{\frac{k_{B} T m}{\pi}} \frac{f_{\eta}\left(T^{*}\right)}{\sigma^{2} \Omega^{(2.2)^{*}}\left(T^{*}\right)}
$$

where $T^{*}=T / \varepsilon$ when $\mathrm{T}$ and $\varepsilon$ are both expressed in $\mathrm{K}$ and $f_{\eta}$ is a correction factor associated with the second order solution of the kinetic theory of gases and is calculated as follows:

$$
\begin{aligned}
& f_{\eta}\left(T^{*}\right)=1+\frac{3}{196}\left(8 E^{*}\left(T^{*}\right)-7\right)^{2} \\
& E^{*}\left(T^{*}\right)=\frac{\Omega^{(2.3)^{*}}\left(T^{*}\right)}{\Omega^{(2.2)^{*}}\left(T^{*}\right)}
\end{aligned}
$$

where $m$ is the mass of the gas molecule; and $\Omega^{(2,2)^{*}}$ and $\Omega^{(2,3)^{*}}$ are reduced collision integrals [19] that contain all the information of the binary collision dynamics. They are normalized by the cross sections for rigid spheres of diameter $\sigma$. The different collision integrals correspond to variously weighted, energy-averaged cross-sections [20], and they depend on the temperature and the intermolecular potential $\varphi()$.

For low density gases, viscosity and diffusion only depend on elastic collisions [18]. In contrast, thermal conductivity of molecular gases depends on inelastic collisions, and this dependence is approximated using a number of approaches, often involving a Eucken correction [21]. 
The collision diameter $\sigma$ and the well depth $\varepsilon$ can be evaluated using different approaches, including ab initio calculations of potential energy surfaces, scattering measurements, spectroscopic measurements, or determining values that provide the best fit to experiments that measure bulk properties, including transport properties. The latter approach is used here.

Mason and Uribe (1996) [18] give a series expansion to compute collision integrals that they determined empirically by fitting to viscosity measurements for argon. They obtained for $\Omega^{(2,2)^{*}}$ :

$$
\Omega^{(2.2)^{*}}\left(T^{*}\right)=\exp \left[\sum_{i=0}^{4} a_{i}\left(\ln T^{*}\right)^{i}\right]
$$

The collision integral $\Omega^{(2,3)^{*}}$ is computed using the following recursion formula:

$$
\Omega^{(l . s+1)^{*}}\left(T^{*}\right)=\Omega^{(l . s)^{*}}\left(T^{*}\right)+\frac{T}{s+2} \frac{d \Omega^{(l . s)^{*}}}{d T^{*}}\left(T^{*}\right)
$$

yielding :

$$
\Omega^{(2.3)^{*}}\left(T^{*}\right)=\Omega^{(2.2)^{*}}\left(T^{*}\right) \cdot\left(1+\frac{1}{4}\right) \exp \left[\sum_{i=1}^{4} i a_{i}\left(\ln T^{*}\right)^{i-1}\right]
$$

The $a_{i}$ 's are given in Table II. The expressions given here for the collision integrals are valid only for $1<T^{*}<10$, for temperatures outside this range refer to Mason et al. [18]. These collision integrals are very similar to those tabulated in Hirschfelder et al. [19], and those given in TRANLIB for non-polar species, for which tabulated results are from Monchick and Mason [22]. Another common approach to calculating collision integrals is to assume a given functional form of the intermolecular potential, and numerically evaluate the integrals. Many different models have been proposed for the intermolecular potential. The Lennard-Jones 12-6 potential $\varphi$ is used throughout the present study, and is:

$$
\varphi(r)=4 \varepsilon\left[\left(\frac{\sigma}{r}\right)^{12}-\left(\frac{\sigma}{r}\right)^{6}\right]
$$

Calculation of the collision integrals with this potential yields a curve quite close to the empirically determined curve of Mason and Uribe, as shown in Figure 1; they agree within 1\% 
for almost the entire range of $T^{*}$ shown. We approximate $\Omega^{(2.2)^{*}}$ for the Lennard-Jones potential using the same four-term series expansion as Equation (4); results are shown in Table II.

\section{III - Non uniqueness of the potential parameters}

Mourits and Rummens [4] review intermolecular potentials based upon viscosity measurements, and point out that many combinations of the potential parameters, $\varepsilon_{\mathrm{i}}$ and $\sigma_{\mathrm{i}}$, can be used to calculate viscosities of acceptable precision ( $2 \%$ or less) for species i. The nonuniqueness or indeterminacy of the potential parameters has not been recognized in many disciplines like the combustion and chemical physics communities that use transport properties in various applications.

When the temperature is fixed, there is a curve in $(\varepsilon, \sigma)$ along which the viscosity is constant. From equation (1), if the parameter pair $\left(\varepsilon_{0}, \sigma_{0}\right)$ generates a viscosity prediction that matches experiment, then another $(\varepsilon, \sigma)$ will predict the same viscosity if

$$
\sigma^{2} \Omega^{(2,2) *}(T / \mathcal{E})=\sigma_{0}^{2} \Omega^{(2,2)^{*}}\left(T / \varepsilon_{0}\right)
$$

where the correction factor $f_{\eta}$ is ignored since it is close to unity.

Kim and Ross determined the shape of the curve for some special cases of reduced temperature for which analytical approximations for the collision integral are available; for instance, for low temperature $\left(0.4<\mathrm{T}^{*}<1\right)$, the curve is defined by

$$
\sigma \propto \frac{c}{\sqrt[4]{\varepsilon}}
$$

where $\mathrm{c}$ is a constant; with this expression, any $(\varepsilon, \sigma)$ that provides a good fit to data can be used to determine the curve, within the range of $\mathrm{T}$ and $\varepsilon$ for which $\mathrm{T}^{*}$ is in the designated range. Kim and Ross suggest an approximation to the collision integral for $1<\mathrm{T}^{*}<5$, from which we derive the relationship 


$$
\sigma=\sigma_{0} \sqrt{\frac{k T+1.09 \varepsilon_{0}}{k T+1.09 \varepsilon}}
$$

Outside the range of $\mathrm{T}^{*}$ encompassed by the above equations, Equation (8) can be solved numerically for $\sigma$ as a function of $\varepsilon$ to generate a curve in $(\varepsilon, \sigma)$ space such that the calculated viscosity at a given temperature is the same everywhere along the curve. For instance, Figure 2 shows $\Omega^{(2.2)^{*}}$ versus $\mathrm{T}^{*}$, with two arbitrary points identified. By choosing appropriate $\sigma$ values, either point shown generates the same value of viscosity. The resulting curve is shown in Figure 3 for $\mathrm{T}=200,400$, and $600 \mathrm{~K}$, for two $\left(\varepsilon_{0}, \sigma_{0}\right)$ pairs, corresponding to Ar and $\mathrm{CH}_{4}$.

Higher $T$ curves have smaller slopes as shown in Figure 3; and this is indicative of declining sensitivity of the collision integrals with respect to the well depth with temperature. In highenergy ( $>k_{B} T$ ) collisions the contribution to the collision integrals is dominated by collisions with impact parameters comparable to or less than $\sigma$, which have a distance of closest approach in the repulsive region of the potential. The potential well has relatively little influence on these collisions. In low-energy collisions, the potential well affects the trajectories (and therefore the collision integrals), and the contribution to the collision integrals is dominated by collisions with impact parameters greater than $\sigma$, most of which do not sample the repulsive region of the potential. At high $T$, most collisions are of high energy, so a change in the depth of the potential well has little influence on the collision integrals; hence even a large increase in the depth of the well can be offset by a small decrease in the size of the repulsive region. Thus the hightemperature curves in Figure 3 have a shallow slope. At low $T$, on the other hand, most collisions are of low energy and are sensitive to the well depth. Compared to the high- $T$ case, an increase in the depth of the potential well requires a larger change in the size of the repulsive region to generate the same collision integrals. 
The existence of a curve in $(\varepsilon, \sigma)$ along which the viscosity is constant at a given $T$ has important practical implications in determining parameter values from viscosity data by comparing viscosity calculated from Equation (1) to a set of $\mathrm{N}_{\exp }$ experimental values. Since each temperature has a different curve, no single curve will predict viscosity perfectly over a wide range of temperatures. Curves for different temperatures lie very close to one another; hence, a single curve can work well enough to match data within experimental error over a range of temperatures as we show below.

The absolute mean relative error, $\Delta$, is defined as follows:

$$
\Delta=\frac{1}{N_{\exp }} \sum_{k=1}^{N_{\exp }} \frac{\left|\eta_{k}^{\text {calc }}-\eta_{k}^{\exp }\right|}{\eta_{k}^{\exp }}
$$

We use the absolute mean relative error, rather than (for instance) the root-mean-squared error, to decrease the influence of outliers. Fitting consists of finding values of $\sigma$ and $\varepsilon$ such that $\Delta$ is minimized. Since there are only two parameters, minimizing $\Delta$ can be done quickly by calculating $\Delta$ on a grid of $\sigma$ and $\varepsilon$ and refining the grid near the minima. The Powell downhill method and simulated annealing have also been used but they are more complicated and timeconsuming to implement, and produced results identical to the grid approach.

The fitting of pure species parameters is considered first. The function $\Delta$ versus $\sigma$ and $\varepsilon$ is shown in Figure 4, and has the same shape for all the systems that have been investigated. As indicated in Figure 3, there is a single $(\sigma, \varepsilon)$ pair at which $\Delta$ is minimized. A nearly linear curve in the $\sigma, \varepsilon$ plane, which we refer to as a "trough," defines $\sigma, \varepsilon$ pairs that predict the mixture viscosity within $1 \%$ or less. This is comparable in magnitude to the experimental uncertainty in many cases. Moving along the trough toward lower $\varepsilon$, away from the point of best fit, will lead to good estimates of viscosity at an intermediate temperature, but will overestimate the viscosity at 
low $T$, and underestimate it at high $T$. The situation is reversed when moving along the trough towards higher $\varepsilon$. Parameters that characterize the trough for various chemical species are given in Table III, along with the optimal values of the potential parameters obtained through fitting, and values for the parameters tabulated in TRANLIB and MKC.

Figure 5 represents some of the troughs associated with pure species systems listed in Table III, as well as the optimum fitted parameters and those from TRANLIB and MKC. The "fitted parameter" point shows where $\Delta$ is minimized. Values of $\varepsilon$ and $\sigma$ from both the TRANLIB and MKC databases agree well with the values that best fit the experimental data. The parameters tabulated in TRANLIB are slightly off the troughs, while the MKC parameter pairs fall almost perfectly on the troughs. The existence of the trough helps to explain why researchers can use different choices of $(\varepsilon, \sigma)$ for the same species, and still obtain good fits to data.

The optimal $(\varepsilon, \sigma)$ couple was determined by finding the values that minimize the absolute mean relative error over data from several sources. Viscosity measurements by some researchers differ systematically from others; for instance, some researchers report slightly higher viscosity at a given temperature than do others. Thus the optimal parameters are not necessarily those that produce the best fit to the actual viscosities: since different experiments produce different measurements, the actual viscosities are not known. To gauge both the uncertainty in $(\varepsilon, \sigma)$ that is attributable to systematic errors in the viscosity measurements, and the extent to which the details of the trough depend on these errors, the following procedure was followed. Optimal values of $(\varepsilon$, $\sigma$ ) and a description of the trough were determined for several different datasets, for $\mathrm{Ar}, \mathrm{CH}_{4}$ and $\mathrm{N}_{2}$. For each species, data from a single source were excluded and the optimal values and the 
equation for the trough were determined for the remaining data; then data from a different single source were excluded; and so on.

In the case of $\mathrm{N}_{2}$, excluding data from any single source made little difference to the best-fit $\varepsilon$ or $\sigma$, or the trough: the largest $\varepsilon$ differed from the smallest by only $3 \%$, and the largest $\sigma$ differed from the smallest by less than $0.3 \% . \mathrm{CH}_{4}$ generated more variability, with a $4 \%$ difference between the highest and the lowest $\varepsilon$ and a $0.5 \%$ variation in $\sigma$. Ar had a $6 \%$ variation in $\varepsilon$ and a $0.6 \%$ variation in $\sigma$. For the three species, when viscosity data from a single source are excluded, the resulting $(\varepsilon, \sigma)$ couple falls very close to the trough generated from the full dataset. In summary, although there is some uncertainty in $\varepsilon$ and $\sigma$ there is little uncertainty in the location and shape of the trough in $(\varepsilon, \sigma)$ space.

It is also of interest to consider how the error in the viscosity calculations varies with temperature, and this is shown for $\mathrm{N}_{2}$ in Figure 6. The different sets of potential parameters used are presented in Table IV. The parameters we call Set A and Set B are chosen to be on the trough, but with well depths that differ by $\pm 15 \%$ from the best-fit values. The corresponding collision diameters are computed using the slope of the line representing the trough.

With a difference in the well depths of $30 \%$ compensated by a roughly $3 \%$ difference in the collision diameters, we obtain predictions that agree with experiment to within the experimental uncertainty of less than $1 \%$ for a large range of temperatures (300 to $1000 \mathrm{~K})$. In contrast, using TRANLIB parameters that are individually closer to the fitted parameters - but that, considered as an $(\varepsilon, \sigma)$ pair, are slightly off the trough - results in deviations between 1 and $2 \%$, outside the experimental uncertainty range. 
Figure 6 shows deviations of estimated viscosity from experimental measurements for $\mathrm{N}_{2}$ for several sets of measurements. Each experimental measurement generates five points on Figure $6 \mathrm{~b}$, since each measurement is compared to the viscosity calculated using five different parameter pairs.

\section{IV - Combining rules}

The viscosity of a mixture of $v$ components $\eta_{\operatorname{mix}}$ is given by the kinetic theory of gases in first order as [12]:

$$
\eta_{\text {mix }}=\frac{\left|\begin{array}{ccccc}
H_{11}^{00} & H_{12}^{00} & \cdots & H_{1 v}^{00} & x_{1} \\
H_{21}^{00} & H_{22}^{00} & \cdots & H_{2 v}^{00} & x_{2} \\
\vdots & \vdots & \ddots & \vdots & \vdots \\
H_{v 1}^{00} & H_{v 2}^{00} & \cdots & H_{v v}^{00} & x_{v} \\
x_{1} & x_{2} & \cdots & x_{v} & 0
\end{array}\right|}{\left|\begin{array}{cccc}
H_{11}^{00} & H_{12}^{00} & \cdots & H_{1 v}^{00} \\
H_{21}^{00} & H_{22}^{00} & \cdots & H_{2 v}^{00} \\
\vdots & \vdots & \ddots & \vdots \\
H_{v 1}^{00} & H_{v 2}^{00} & \cdots & H_{v v}^{00}
\end{array}\right|}
$$

where:

$$
\begin{aligned}
& H_{i i}^{00}=\frac{x_{i}^{2}}{\eta_{i}}+\sum_{\substack{k=1 \\
k \neq i}}^{v} \frac{2 x_{i} x_{k}}{\eta_{i k}} \frac{m_{i} m_{k}}{\left(m_{i}+m_{k}\right)^{2}}\left(\frac{5}{3 A_{i k}^{*}\left(T^{*}\right)}+\frac{m_{k}}{m_{i}}\right) \\
& H_{i j}^{00}=-\frac{2 x_{i} x_{j}}{\eta_{i j}} \frac{m_{i} m_{j}}{\left(m_{i}+m_{j}\right)^{2}}\left(\frac{5}{3 A_{i j}^{*}\left(T^{*}\right)}-1\right) \\
& A_{i j}^{*}\left(T^{*}\right)=\frac{\Omega_{i j}^{(2,2)^{*}}\left(T^{*}\right)}{\Omega_{i j}^{(1,1)^{*}}\left(T^{*}\right)}
\end{aligned}
$$

where $\Omega_{i j}^{(l, s)^{*}}\left(T^{*}\right)=\Omega^{(l, s)^{*}}\left(T / \varepsilon_{i j}\right)$.

$\eta_{\mathrm{ij}}$ is the viscosity of a hypothetical gas whose interactions are described by the parameters $\sigma_{\mathrm{ij}}$ and $\varepsilon_{\mathrm{ij}}$, and whose mass is twice the reduced mass of the two interacting species. $\mathrm{x}_{\mathrm{i}}$ is the mole fraction of species $i$. 
$\Omega^{(1,1)^{* *}}$ is computed using the expression given by Mason and Uribe (1996) [18]:

$$
\Omega^{(1.1)^{*}}\left(T^{*}\right)=\exp \left[\sum_{i=0}^{4} b_{i}\left(\ln T^{*}\right)^{i}\right]
$$

The coefficients $b_{i}$ 's are given in Table II.

The potential parameters $\sigma_{\mathrm{ij}}$ and $\varepsilon_{\mathrm{ij}}$ can be determined with several approaches, including finding the values that provide the best fit to experimental data, or using "combining rules" to predict them from $\sigma$ and $\varepsilon$ for the individual species as described below. Determining potential parameters by fitting requires a rich and accurate experimental data set. Combining rules are essential to predicting scaling parameters for interactions for which there is inadequate experimental data.

Combining rules predict the potential parameters characterizing the interactions of two unlike molecules from parameters characterizing each of the individual molecules. The most commonly used combining rule for the collision diameter is the arithmetic mean (AM):

$$
\sigma_{i j}=\frac{\sigma_{i i}+\sigma_{j j}}{2}
$$

This rule is exact if the interacting molecules are hard spheres. The most commonly used combining rule for the well depth is the geometric mean (GM), also called the Berthelot rule:

$$
\varepsilon_{i j}=\sqrt{\varepsilon_{i i} \varepsilon_{j j}}
$$

Other rules have been proposed which give more weight to the component with the weaker intermolecular forces [12]. One of these is the harmonic mean (HM), originally advocated by Fender and Halsey (1962) [23]:

$$
\varepsilon_{i j}=\frac{2 \varepsilon_{i i} \varepsilon_{j j}}{\varepsilon_{i i}+\varepsilon_{j j}}
$$

More sophisticated combining rules like that of Kong (1973) [24] have been proposed for $\sigma_{\mathrm{ij}}$ and $\varepsilon_{\mathrm{ij}}$ : 


$$
\begin{aligned}
& \sigma_{i j}=\frac{\left[\left(\varepsilon_{i i} \sigma_{i i}^{12}\right)^{1 / 13}+\left(\varepsilon_{j j} \sigma_{j j}^{12}\right)^{1 / 13}\right]^{13 / 6}}{2^{13 / 6}\left(\varepsilon_{i i} \varepsilon_{j j}\right)^{1 / 12}\left(\sigma_{i i} \sigma_{j j}\right)^{1 / 2}} \\
& \varepsilon_{i j}=\frac{\varepsilon_{i i} \varepsilon_{j j} \sigma_{i i}^{6} \sigma_{j j}^{6}}{\left(\varepsilon_{i i} \sigma_{i i}^{12}\right) / 2^{13} \cdot\left\{1+\left[\left(\varepsilon_{j j} \sigma_{j j}^{12}\right) /\left(\varepsilon_{i i} \sigma_{i i}^{12}\right)\right]^{1 / 13}\right\}^{13}}
\end{aligned}
$$

Equations (20) and (21) are specifically for a Lennard-Jones 12-6 potential as specified in reference [12].

\section{V- Combined parameters obtained from fitting to viscosity data.}

The same basic approach that was used for pure species is used to fit $\sigma_{\mathrm{ij}}$ and $\varepsilon_{\mathrm{ij}} \mathrm{using}$ experimental data reported in Kestin et al. [3] The parameters $\left(\varepsilon_{\mathrm{ii}}, \sigma_{\mathrm{ii}}\right)$ and $\left(\varepsilon_{\mathrm{jj}}, \sigma_{\mathrm{jj}}\right)$ were determined from the best fit to pure species viscosities, and the combined parameters $\sigma_{\mathrm{ij}}$ and $\varepsilon_{\mathrm{ij}}$ are obtained by finding the values that, when used in Equation (12), yield the best fit to experimental data associated with the binary mixture. As with the pure species, a trough, approximated by a straight line determined by linear regression, was found for each mixture.

Table V contains the fitted values, the parameter values obtained from different combining rules, and the slope of the line representing the trough.

Different combining rules give different values for $\sigma_{\mathrm{ij}}$ and $\varepsilon_{\mathrm{ij}}$, and for some mixtures the differences are rather large, as illustrated in Figure 7, which shows $\sigma_{\mathrm{O} 2-\mathrm{X}}$ (where $\mathrm{X}=\mathrm{CF}_{4}, \mathrm{SF}_{6}$, and $\mathrm{CO}_{2}$ ) versus $\sigma_{\mathrm{X}-\mathrm{X}}$ as well as $\varepsilon_{\mathrm{O} 2-\mathrm{X}}$ versus $\varepsilon_{\mathrm{X}-\mathrm{X}}$. Figure 8 shows how the results of the combining rules relate to the trough for some mixtures. Although yielding very different values, the various combining rules produce couples of parameters $\varepsilon_{i j}, \sigma_{i j}$ that lie close to the trough line and therefore generate predicted mixture viscosities in reasonable agreement with experiment. Viscosities calculated using parameters obtained with standard combining rules tend to underpredict the viscosity, often by about $1 \%$ to $3 \%$, as shown in Figure 9 . Kong's rule generates 
viscosities that are closer to experimental values than do the other rules in 10 out of the 12 mixtures for which we have data. The agreement between calculated and experimentally determined viscosity is frequently less satisfactory for mixtures than for pure species. The accuracy of the calculations also depends on the composition of the binary mixture as shown in Figure 9 since the concentration of a species acts as a weighting factor for the pure species parameters.

Compared to the fitted values, the arithmetic mean overpredicts the collision diameter. Kong's rule overestimates it even more. Kong's rule, however, underestimates the combined $\varepsilon_{\mathrm{ij}}$, thus yielding $(\varepsilon, \sigma)$ a pair on or near the trough. No consistent over- or under-prediction is observed for the geometric mean or the harmonic mean.

In many applications, the geometric mean and harmonic mean rules for the well depth are used with the arithmetic mean for collision diameter. TRANLIB and MKC use the arithmetic mean for collision diameters for non-polar species; TRANLIB uses the geometric mean for the well depth and MKC adds a correction factor to it, following the recommendations of Bzowski et al. $[13,17]$ and Tang and Toennies [25].

\section{1) Combined collision diameters}

Plotting the $\sigma_{\mathrm{ij}}$ 's versus the pure species collision diameters $\sigma_{\mathrm{ii}}$ 's and $\sigma_{\mathrm{jj}}$ 's results in a nearly planar surface as shown in Figure 10. The equation of the plane yielding the best fit in terms of absolute mean relative error is:

$$
\sigma_{i j}=0.43 \cdot\left(\sigma_{i i}+\sigma_{j j}\right)+0.49
$$

Results are presented in Table VI and Figure 10. Equation (22) yields good results with the small dataset that has been investigated, with all deviations less than $2.5 \%$ from the best-fit values, frequently less than $2 \%$, except for one outlier $\left(\mathrm{SF}_{6}-\mathrm{SF}_{6}\right)$, for which the deviation is $4 \%$. 
Equation (22) is empirical, but is similar to the arithmetic mean rule that would apply if molecules were hard spheres. In the case $\sigma_{i i}=\sigma_{j j}$, one might expect $\sigma_{i j}=\sigma_{i i}=\sigma_{j j}$, which is not the case for Equation (22). But in fact, if we imagine a case in which two different species have the same collision diameters, there is no physical requirement that the effective collision diameter for the interaction between them must be the same as when each interacts with a molecule of its own type. Notably, Kong's Rule (Equation (21)) recognizes this fact.

No simple function of $\varepsilon_{\mathrm{ii}}$ and $\varepsilon_{\mathrm{jj}}$ alone provides a good fit to the data in Table V.

\section{V - Conclusions}

The Law of Corresponding States has been demonstrated for a number of pure substances and binary mixtures, and provides evidence that the transport properties viscosity and diffusion can be determined from a molecular shape function, often taken to be a Lennard-Jones 12-6 potential that requires two scaling parameters: a well depth $\varepsilon_{\mathrm{ij}}$ and a collision diameter $\sigma_{\mathrm{ij}}$, both of which depend on the interacting species $i$ and $j$. We show that there is a curve in $\left(\varepsilon_{\mathrm{ii}}, \sigma_{\mathrm{ii}}\right)$ space such that parameter values on the curve generate a calculated viscosity in good agreement with measurements for a pure gas. These results help to explain why different researchers sometimes disagree on the values of the parameters, for a given species. We obtain estimates for $\varepsilon_{\mathrm{ij}}$ and $\sigma_{\mathrm{ij}}$ of interacting species by finding the values that provide the best fit to viscosity data for binary mixtures. As with the pure species, we found that there is a curve in $\left(\varepsilon_{\mathrm{ij}}, \sigma_{\mathrm{ij}}\right)$ space such that parameter values on the curve generate a calculated viscosity in good agreement with measurements. The curves in both cases are approximated well with a straight line. We calculated parameters for many binary pairs using four combining rules. Different combining rules give different values for $\sigma_{\mathrm{ij}}$ and $\varepsilon_{\mathrm{ij}}$ and for some mixtures the differences are rather large. 
However, the various combining rules produce couples of parameters $\varepsilon_{\mathrm{ij}}, \sigma_{\mathrm{ij}}$ that lie close to the curve (line) in ( $\left.\varepsilon_{\mathrm{ij},} \sigma_{\mathrm{ij}}\right)$ space and therefore generate predicted mixture viscosities in satisfactory agreement with experiment. Kong's rule was found to work better than other combining rules for predicting viscosity, but none of the combining rules consistently yields parameter values near the best-fit values, suggesting that improved rules could be developed. We presented empirical rules that work well for a small dataset.

\section{Acknowledgement}

The author gratefully acknowledge by the Director, Office of Science, Office of Basic Energy

Sciences, Chemical Sciences, Geosciences, and Biosciences Division of the U.S., Department of Energy, under contract No. DE-AC02-05CH11231. 


\section{VI - Bibliography}

1. Brown, N. J.; Munn, R. J. J Chem Phys 1972, 57, 2216-2218.

2. Wakeham, W. A.; Assael, M. A.; Atkinson, J. K.; Bilek, J.; Fareleira, J. M. N. A.; Fitt, A. D.; Goodwin, A. R. H.; Oliveira, C. M. B. P. Int J Thermophys 2007, 28, 372-416.

3. Kestin, J.; Khalifa, H. E.; Ro, S. T.; Wakeham, W. A. Physica A 1977, 88, 242-260.

4. Mourits, F. M.; Rummens, F. H. A. Can J Chem 1977, 15, 3007-3020.

5. Wang, H.; Law, C. K. Diffusion Coefficient of Hydrogen Atom for Combustion Modeling. Technical Meeting of the Eastern States Section of the Combustion Institute: Hilton Head, SC; 1996.

6. Paul, P. H. DRFM, A New Package for the Evaluation of Gas-Phase-Transport Properties. SAND98-8203 UC-1409: SANDIA National Laboratories; 1997.

7. Paul, P. H.; Warnatz, J. Proc Combust Inst 1998, 27, 495-504.

8. Mehdipour, N.; Eslami, H. Int J Therm Sci 2002, 41, 949-954.

9. Cappelletti, D.; Vecchiocattivi, F.; Pirani, F.; Heck, E. L.; Dickinson, A. S. Mol Phys 1998, 93, 485-499.

10. Kim, S. K.; Ross, J. J Chem Phys 1967, 46, 818.

11. Barker, J. A.; Pompe, A. Aust J Chem 1968, 21, 1683-1694.

12. Maitland, G. C.; Rigby, M.; Smith, E. B.; Wakeham, W. A. Intermolecular Forces: Their Origin and Determination. Clarendon Press: Oxford; 1981.

13. Bzowski, J.; Mason, E. A.; Kestin, J. Int J Thermophys 1988, 9, 131-143.

14. Kee, R. J.; Dixon-Lewis, G.; Warnatz, J.; Coltrin, M. E.; Miller, J. A. A FORTRAN Computer Code Package for the Evaluation of Gas-Phase Multicomponent Transport Properties. SANDIA: ; 1986.

15. Najafi, B.; Mason, E. A.; Kestin, J. Physica A 1983, 119, 387-440.

16. Boushehri, A.; Bzowski, J.; Kestin, J.; Mason, E. A. J Phys Chem Ref Data 1987, 16, 445-466.

17. Bzowski, J.; Kestin, J.; Mason, E. A.; Uribe, F. J. J Phys Chem Ref Data 1990, 19, 1179-1232.

18. Mason, E. A.; Uribe, F. J. In Transport Properties of Fluids: Their Correlation, Prediction, and Estimation, 1st ed.; Millat, J.; Dymond, J. H.; Nieto de Castro, C. A., Ed.; Cambridge University Press: Cambridge, 1996; p. 250-282.

19. Hirschfelder, J. O.; Curtiss, C. F.; Bird, R. B. Molecular Theory of Gases and Liquids. John Wiley and Sons: New York; 1954. 
20. Kestin, J.; Wakeham, W. A. Transport Properties of Fluids - Thermal Conductivity, Viscosity, and Diffusion Coefficient. Hemisphere Publishing Corporation: New York; 1988.

21. Brown, N. J.; Bastien, L. A. J.; Price, P. N. Transport Properties for Combustion Modeling, Submitted to Progress in Energy and Combustion Science, April 2010.

22. Monchick, L.; Mason, E. A. J Chem Phys 1961, 35, 1676-1697.

23. Fender, B. E. F.; Halsey, Jr., G. D. J Chem Phys 1962, 36, 1881-1888.

24. Kong, C. L. J Chem Phys 1973, 59, 968-969.

25. Tang, K. T.; Toennies, J. P. J Chem Phys 1984, 80, 3726-3741.

26. Kestin, J.; Ro, S. T.; Wakeham, W. A. J Chem Phys 1972, 56, 4086-4091.

27. Kalelkar, A. S.; Kestin, J. J Chem Phys 1970, 52, 4248-4261.

28. Kestin, J.; Ro, S. T.; Wakeham, W. A. J Chem Phys 1972, 56, 4119-4124.

29. May, E. F.; Berg, R. F.; Moldover, M. R. Int J Thermophys 2007, 28, 1085-1110.

30. Hellemans, J. M.; Kestin, J.; Ro, S. T. Physica 1973, 65, 376-380.

31. Schley, P.; Jaeschke, M.; Küchenmeister, C.; Vogel, E. Int J Thermophys 2004, 25, 1623-1652.

32. Abe, Y.; Kestin, J.; Khalifa, H. E.; Wakeham, W. A. Physica A 1978, 93, 155-170.

33. Kestin, J.; Ro, S. T.; Wakeham, W. A. J Chem Phys 1972, 56, 4114-4118.

34. Seibt, D.; Vogel, E.; Bich, E.; Buttig, D.; Hassel, E. J Chem Eng Data 2006, 51, 526-533.

35. Kestin, J.; Ro, S. T.; Wakeham, W. A. J Chem Phys 1972, 56, 4036-4042.

36. Hellemans, J. M.; Ro, S. T.; Kestin, J. J Chem Phys 1972, 57, 4038-4042.

37. Hellemans, J. M.; Kestin, J.; Ro, S. T. Physica 1973, 65, 362-375.

38. Kestin, J.; Ro, S. T.; Wakeham, W. A. J Chem Phys 1972, 56, 5837-5842. 


\section{Tables}

Table I: Experimental data used for the fitting of the potential parameters

Table II. Numerical coefficients for the calculation of the reduced collision integrals $\Omega^{(1,1)^{*}}, \Omega^{(2,2)^{* *}}$, and $\Omega^{(2,3)^{* *}}$. Table III. Fitted potential parameters, potential parameters given in TRANLIB and MKC, and numerical characterization of the trough for different pure species systems.

Table IV. Potential parameters for $\mathrm{N}_{2}$ obtained different ways and used in the computations presented in Figure 6. Table V. Fitted potential parameters, potential parameters obtained from different combining rules, and the slope of the line representing the trough for twelve different binary mixture systems.

Table VI. Collision diameters fit by a plane. 
Table I: Experimental data used for the fitting of the potential parameters

\begin{tabular}{cc}
\hline \hline System & References \\
\hline $\mathrm{Ar}$ & {$[3,26-29]$} \\
$\mathrm{CH}_{4}$ & {$[3,29-32]$} \\
$\mathrm{CO}_{2}$ & {$[3,33]$} \\
$\mathrm{N}_{2}$ & {$[3,34-38]$} \\
$\mathrm{O}_{2}$ & {$[37]$} \\
$\mathrm{CF}_{4}$ & {$[3]$} \\
$\mathrm{SF}_{6}$ & {$[3]$} \\
Binary Mixtures & {$[3]$} \\
\hline \hline
\end{tabular}


Table II. Numerical coefficients for the calculation of the reduced collision integrals $\Omega^{(1,1)^{*}}$, $\Omega^{(2,2)^{*}}$, and $\Omega^{(2,3)^{*}}$.

\begin{tabular}{ccccc}
\hline \hline$i$ & $a_{i}^{\text {Lennard }-J o n e s}$ & $a_{i}$ & $b_{i}$ (Rare gases) & $b_{i}$ (Polyatomic gases) \\
\hline 0 & 0.4729 & 0.46641 & 0.357588 & 0.295402 \\
1 & -0.5693 & -0.56991 & -0.472513 & -0.510069 \\
2 & 0.1995 & 0.19591 & 0.0700902 & 0.189395 \\
3 & -0.0407 & -0.03879 & 0.016574 & -0.045427 \\
4 & 0.0030 & 0.00259 & -0.00592022 & 0.0037928 \\
\hline \hline
\end{tabular}


Table III. Fitted potential parameters, potential parameters given in TRANLIB and MKC, and numerical characterization of the trough for different pure species systems.

\begin{tabular}{cccccccc}
\cline { 2 - 6 } & \multicolumn{3}{c|}{ Well depth $\varepsilon(\mathrm{K})$} & \multicolumn{3}{c|}{ Collision Diameter $\sigma(\AA)$} & \\
\hline Species & $\begin{array}{c}\text { This } \\
\text { work }\end{array}$ & TRANLIB & MKC & $\begin{array}{c}\text { This } \\
\text { work }\end{array}$ & TRANLIB & MKC & $\begin{array}{c}\text { Slope } \\
\left(\mathrm{m}^{\prime} \AA \mathrm{K}^{-1}\right)\end{array}$ \\
\hline $\mathrm{Ar}$ & 143.0 & 136.500 & 141.5 & 3.35 & 3.330 & 3.350 & -3.45 \\
$\mathrm{CH}_{4}$ & 165.3 & 141.400 & 161.4 & 3.71 & 3.746 & 3.721 & -4.20 \\
$\mathrm{CO}_{2}$ & 249.8 & 244.000 & 245.3 & 3.76 & 3.763 & 3.769 & -2.78 \\
$\mathrm{~N}_{2}$ & 102.4 & 97.530 & 98.4 & 3.64 & 3.621 & 3.652 & -3.83 \\
$\mathrm{O}_{2}$ & 127.9 & 107.400 & 121.1 & 3.38 & 3.458 & 3.407 & -3.75 \\
$\mathrm{CF}_{4}$ & 160.0 & $x$ & 156.5 & 4.56 & x & 4.579 & -3.98 \\
$\mathrm{SF}_{6}$ & 197.8 & x & 207.7 & 5.30 & x & 5.252 & -4.92 \\
\hline \hline
\end{tabular}


Table IV. Potential parameters for $\mathrm{N}_{2}$ obtained different ways and used in the computations presented in Figure 6.

\begin{tabular}{ccc}
\hline \hline Source & $\varepsilon(\mathrm{K})$ & $\sigma(\AA)$ \\
\hline Fit & 102.4 & 3.64 \\
TRANLIB & 97.530 & 3.621 \\
MKC & 98.40 & 3.652 \\
On trough at A & 87.0 & 3.70 \\
On trough at B & 117.8 & 3.58 \\
\hline \hline
\end{tabular}


Table V. Fitted potential parameters, potential parameters obtained from different combining rules, and the slope of the line representing the trough for twelve different binary mixture systems.

GM: Geometric Mean. HM: Harmonic Mean. AM: Arithmetic Mean. Kong: Kong's rule.

\begin{tabular}{|c|c|c|c|c|c|c|c|c|}
\hline & \multicolumn{4}{|c|}{ Well depth $\varepsilon(\mathrm{K})$} & \multicolumn{3}{|c|}{ Collision Diameter $\sigma(\AA)$} & \multirow[b]{2}{*}{$\begin{array}{c}\text { Slope } \\
\left(\mathrm{m} \AA . \mathrm{K}^{-1}\right)\end{array}$} \\
\hline $\begin{array}{l}\text { Binary } \\
\text { Mixture }\end{array}$ & $\begin{array}{l}\text { This } \\
\text { work }\end{array}$ & GM & $\mathrm{HM}$ & Kong & $\begin{array}{l}\text { This } \\
\text { work }\end{array}$ & $\mathrm{AM}$ & Kong & \\
\hline $\mathrm{Ar}-\mathrm{CF}_{4}$ & 142.7 & 151.3 & 151.1 & 131.6 & 3.96 & 3.96 & 4.00 & -3.28 \\
\hline $\mathrm{Ar}-\mathrm{SF}_{6}$ & 183.6 & 168.2 & 166.0 & 121.6 & 4.20 & 4.33 & 4.45 & -3.68 \\
\hline $\mathrm{CF}_{4}-\mathrm{SF}_{6}$ & 209.1 & 178.1 & 177.1 & 171.2 & 4.77 & 4.93 & 4.95 & -4.32 \\
\hline $\mathrm{CH}_{4}-\mathrm{CF}_{4}$ & 160.3 & 162.8 & 162.7 & 153.7 & 4.14 & 4.14 & 4.15 & -3.82 \\
\hline $\mathrm{CH}_{4}-\mathrm{SF}_{6}$ & 175.9 & 180.8 & 180.1 & 149.5 & 4.51 & 4.51 & 4.58 & -4.08 \\
\hline $\mathrm{CO}_{2}-\mathrm{CF}_{4}$ & 188.1 & 200.1 & 195.3 & 193.5 & 4.15 & 4.16 & 4.16 & -3.58 \\
\hline $\mathrm{CO}_{2}-\mathrm{SF}_{6}$ & 235.3 & 222.3 & 220.8 & 192.3 & 4.37 & 4.53 & 4.57 & -3.86 \\
\hline $\mathrm{N}_{2}-\mathrm{CF}_{4}$ & 133.2 & 128.0 & 124.9 & 116.4 & 4.08 & 4.10 & 4.14 & -3.94 \\
\hline $\mathrm{N}_{2}-\mathrm{SF}_{6}$ & 172.1 & 142.3 & 134.9 & 110.3 & 4.30 & 4.47 & 4.58 & -3.69 \\
\hline $\mathrm{O}_{2}-\mathrm{CF}_{4}$ & 152.9 & 143.1 & 142.2 & 124.4 & 3.93 & 3.97 & 4.02 & -3.43 \\
\hline $\mathrm{O}_{2}-\mathrm{CO}_{2}$ & 150.6 & 178.8 & 169.2 & 172.4 & 3.60 & 3.57 & 3.59 & -3.07 \\
\hline $\mathrm{O}_{2}-\mathrm{SF}_{6}$ & 173.0 & 159.1 & 155.4 & 115.0 & 4.24 & 4.34 & 4.47 & -3.79 \\
\hline
\end{tabular}


Table VI. Collision diameters fit by a plane.

\begin{tabular}{ccccccc}
\hline \hline Species 1 & Species 2 & $\sigma_{11}(\AA)$ & $\sigma_{22}(\AA)$ & $\sigma_{12}$ Best fit $(\AA)$ & $\sigma_{12}$ Plane $(\AA)$ & $\begin{array}{c}\text { Deviation } \sigma_{12} \\
\text { Fit-Plane }(\%)\end{array}$ \\
\hline $\mathrm{Ar}$ & $\mathrm{CF}_{4}$ & 3.35 & 4.56 & 3.96 & 3.92 & -1.0 \\
$\mathrm{Ar}$ & $\mathrm{SF}_{6}$ & 3.35 & 5.30 & 4.20 & 4.24 & 1.0 \\
$\mathrm{CF}_{4}$ & $\mathrm{SF}_{6}$ & 4.56 & 5.30 & 4.77 & 4.77 & 0.0 \\
$\mathrm{CH}_{4}$ & $\mathrm{CF}_{4}$ & 3.71 & 4.56 & 4.14 & 4.08 & -1.4 \\
$\mathrm{CH}_{4}$ & $\mathrm{SF}_{6}$ & 3.71 & 5.30 & 4.51 & 4.40 & -2.4 \\
$\mathrm{CO}_{2}$ & $\mathrm{CF}_{4}$ & 3.76 & 4.56 & 4.15 & 4.10 & -1.2 \\
$\mathrm{CO}_{2}$ & $\mathrm{SF}_{6}$ & 3.76 & 5.30 & 4.37 & 4.42 & 1.1 \\
$\mathrm{~N}_{2}$ & $\mathrm{CF}_{4}$ & 3.64 & 4.56 & 4.08 & 4.05 & -0.7 \\
$\mathrm{~N}_{2}$ & $\mathrm{SF}_{6}$ & 3.64 & 5.30 & 4.30 & 4.37 & 1.6 \\
$\mathrm{O}_{2}$ & $\mathrm{CF}_{4}$ & 3.38 & 4.56 & 3.93 & 3.94 & 0.3 \\
$\mathrm{O}_{2}$ & $\mathrm{CO}_{2}$ & 3.38 & 3.76 & 3.60 & 3.59 & -0.3 \\
$\mathrm{O}_{2}$ & $\mathrm{SF}_{6}$ & 3.38 & 5.30 & 4.24 & 4.26 & 0.5 \\
$\mathrm{Ar}$ & $\mathrm{Ar}$ & 3.35 & 3.35 & 3.35 & 3.40 & 1.5 \\
$\mathrm{CF}_{4}$ & $\mathrm{CF}_{4}$ & 4.56 & 4.56 & 4.56 & 4.45 & -2.4 \\
$\mathrm{CH}_{4}$ & $\mathrm{CH}_{4}$ & 3.71 & 3.71 & 3.71 & 3.71 & 0.0 \\
$\mathrm{CO}_{2}$ & $\mathrm{CO}_{2}$ & 3.76 & 3.76 & 3.76 & 3.75 & -0.3 \\
$\mathrm{~N}_{2}$ & $\mathrm{~N}_{2}$ & 3.64 & 3.64 & 3.64 & 3.65 & 0.3 \\
$\mathrm{O}_{2}$ & $\mathrm{O}_{2}$ & 3.38 & 3.38 & 3.38 & 3.42 & 1.2 \\
$\mathrm{SF}_{6}$ & $\mathrm{SF}_{6}$ & 5.30 & 5.30 & 5.30 & 5.09 & -4.0 \\
\hline \hline
\end{tabular}




\section{Figures}

Figure 1. $\Omega^{(2.2)^{*}}$ versus $\mathcal{E}$ for $T=200,400$, and $600 \mathrm{~K}$.

Figure 2. $\Omega^{(2,2)^{*}}$ versus $T^{*}=T / \mathcal{E} \quad$ computed with the 12-6 Lennard-Jones potential.

Figure 3. Curves of $(\varepsilon, \sigma)$ that generate the same predicted viscosity at a given temperature as the pair $\left(\varepsilon_{0}, \sigma_{0}\right)$, shown for two sets of $\left(\varepsilon_{0}, \sigma_{0}\right)$, corresponding to argon and methane for three temperatures: $T=200,400$ (not labeled), and 600K.

Figure 4. Absolute average mean error $\Delta$ between prediction and experiment versus the couple of potential parameter $\sigma, \varepsilon$ for the interaction of $\mathrm{CH}_{4}$ with $\mathrm{CH}_{4}$.

Figure 5. Lines representing the trough for different pure species systems and different sets of potential parameters. Figure 6. (a): Viscosity of $\mathrm{N}_{2}$ versus the temperature $T$ computed with the different couples of potential parameters presented in Table IV and experimental values.

Figure 7. Combined parameters predicted with different combining rules for the interaction of $\mathrm{O}_{2}$ and three other species.

Figure 8. Lines representing the trough for binary mixtures and combined parameters obtained by different methods.

Figure 9. Deviation of the predicted viscosity relative to experimental values versus the temperature, using parameters obtained using different combining rules.

Figure 10. Collision diameters fitted by a plane. 


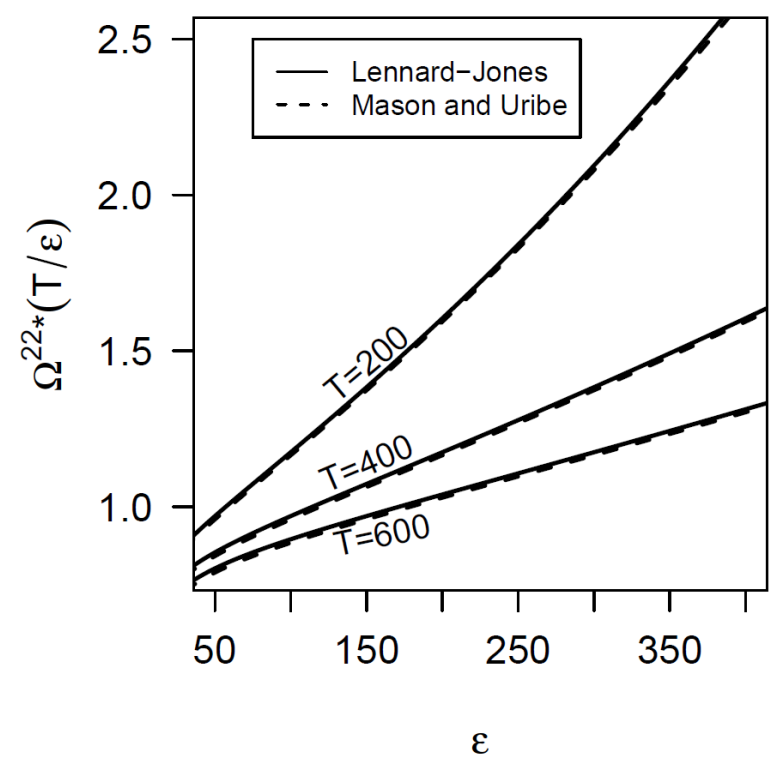

Figure 1. $\Omega^{(2.2)^{*}}$ versus $\varepsilon$ for $\mathrm{T}=200,400$, and $600 \mathrm{~K}$.

Values resulting from the empirical fit of Mason and Uribe are very close to those resulting from a Lennard-Jones potential. The curves are nearly linear over a wide range of $\varepsilon$. 


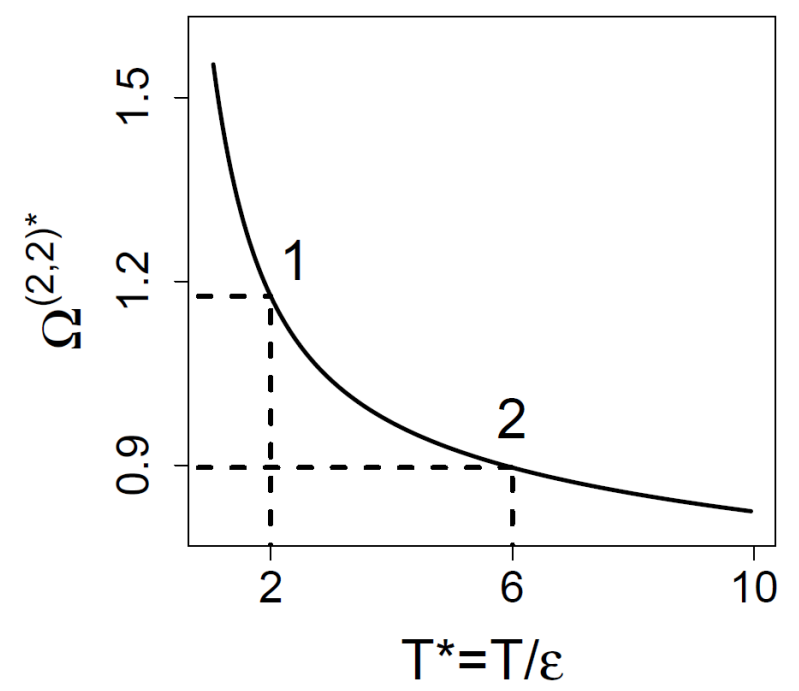

Figure 2. $\Omega^{(2,2)^{*}}$ versus $T^{*}=T / \mathcal{\varepsilon} \quad$ computed with the 12-6 Lennard-Jones potential.

The points 1 and 2 constitute an arbitrary example for which $\sigma_{1}^{2} \Omega^{(2,2)^{*}}\left(T_{1}^{*}\right)=\sigma_{2}^{2} \Omega^{(2,2)^{*}}\left(T_{2}^{*}\right)$. The corresponding values of the parameters for a temperature of $300 \mathrm{~K}$ are $\sigma_{1}=3.00 \AA, \varepsilon_{1}=150.0 \mathrm{~K}$, $\sigma_{2}=3.44 \AA, \varepsilon_{2}=50.0 \mathrm{~K}$, and $\sigma_{1}^{2} \Omega^{(2,2)^{*}}\left(T_{1}^{*}\right)=\sigma_{2}^{2} \Omega^{(2,2)^{*}}\left(T_{2}^{*}\right)=10.58$ 


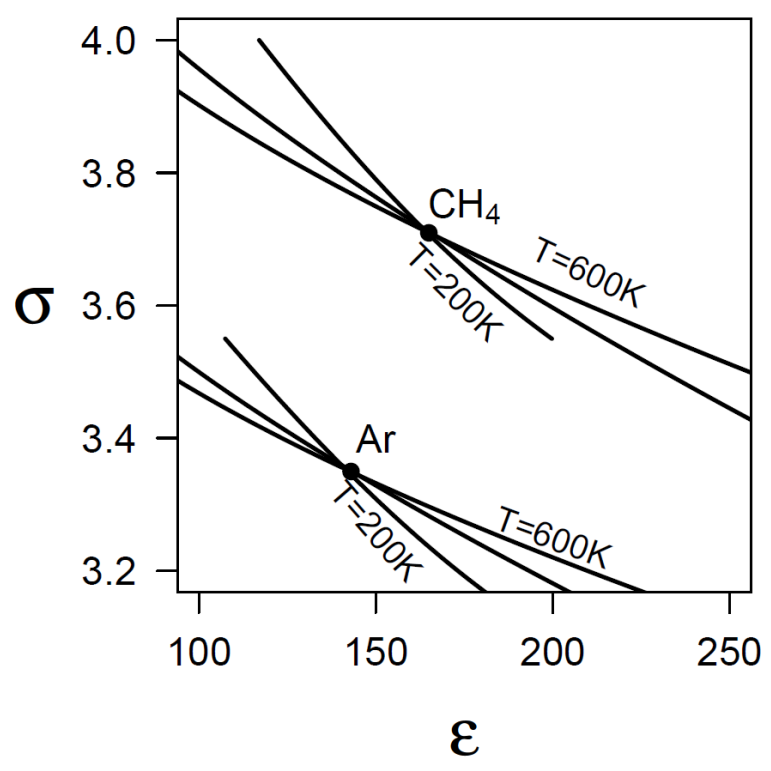

Figure 3. Curves of $(\varepsilon, \sigma)$ that generate the same predicted viscosity at a given temperature as the pair $\left(\varepsilon_{0}, \sigma_{0}\right)$, shown for two sets of $\left(\varepsilon_{0}, \sigma_{0}\right)$, corresponding to argon and methane for three temperatures: $\mathrm{T}=200,400$ (not labeled), and 600K. 


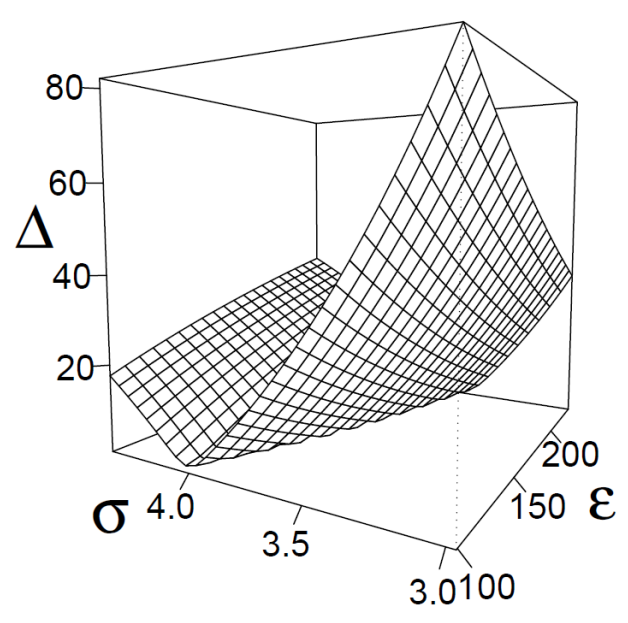

Figure 4. Absolute average mean error $\Delta$ between prediction and experiment versus the couple of potential parameter $\sigma, \varepsilon$ for the interaction of $\mathrm{CH}_{4}$ with $\mathrm{CH}_{4}$. 


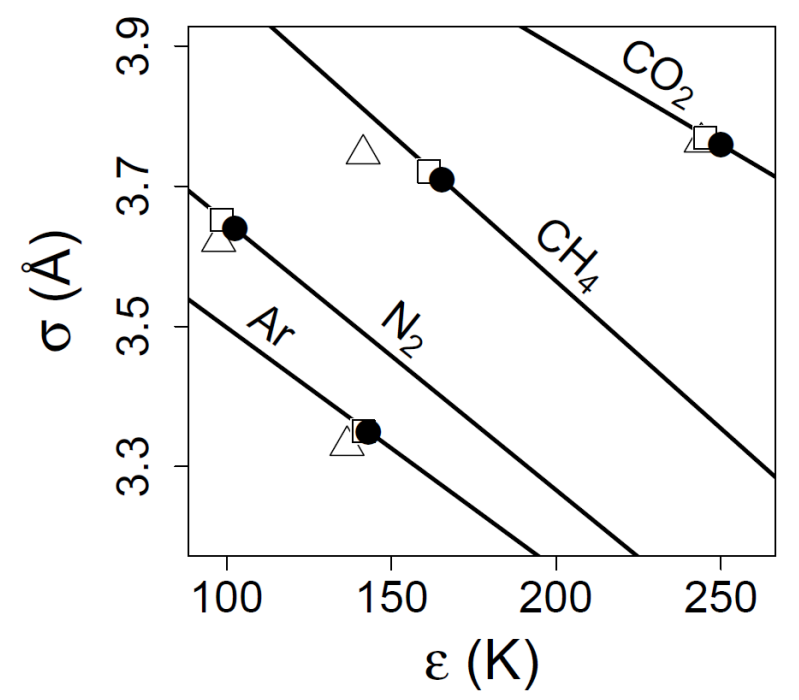

Figure 5. Lines representing the trough for different pure species systems and different sets of potential parameters.

: fitted parameters. $\triangle$ : parameters from TRANLIB. $\square$ : parameters from MKC. The symbols go with the line they are the closest to. 

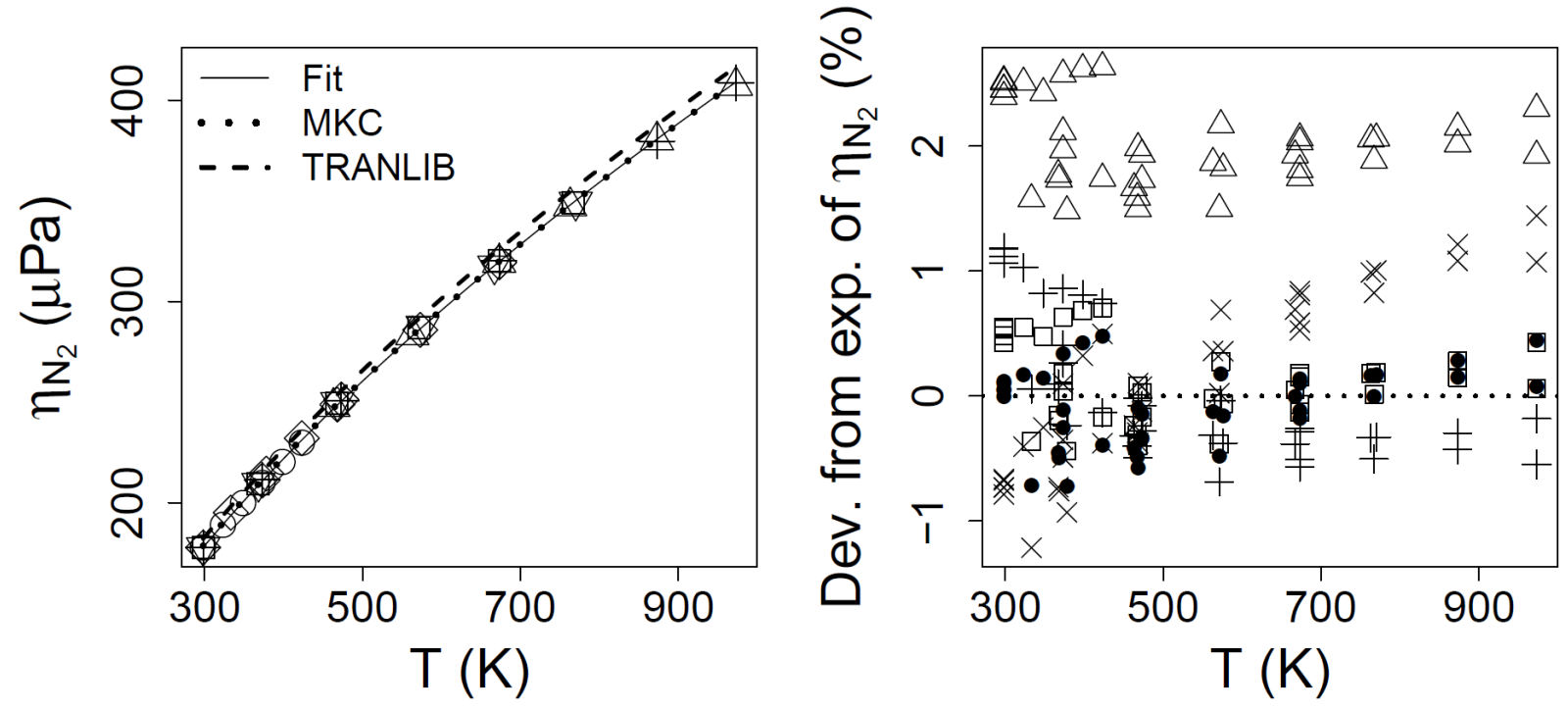

Figure 6. (a): Viscosity of $\mathrm{N}_{2}$ versus the temperature $\mathrm{T}$ computed with the different couples of potential parameters presented in Table IV and experimental values.

O: Seibt et al.(2006), [34] $\triangle$ : Kestin et al (1972),[35] $\diamond:$ Kestin et al. (1977),[3] $\square$ : 1Helleman et al. (1972),[36] $\nabla$ : Helleman et al. (1973),[37] and +: Kestin et al. (1972).[38] (b): Deviation from experiment of predicted viscosity of $\mathrm{N}_{2}$ with the different couples of potential parameters presented in Table IV. The experimental values in (b) are the same as in (a) but the symbols do not identify the experiment, they correspond to the different potential parameters couples used:

: fitted parameters, $\triangle$ : TRANLIB, $\square$ : MKC, + : Set A, $\times$ : Set B. 

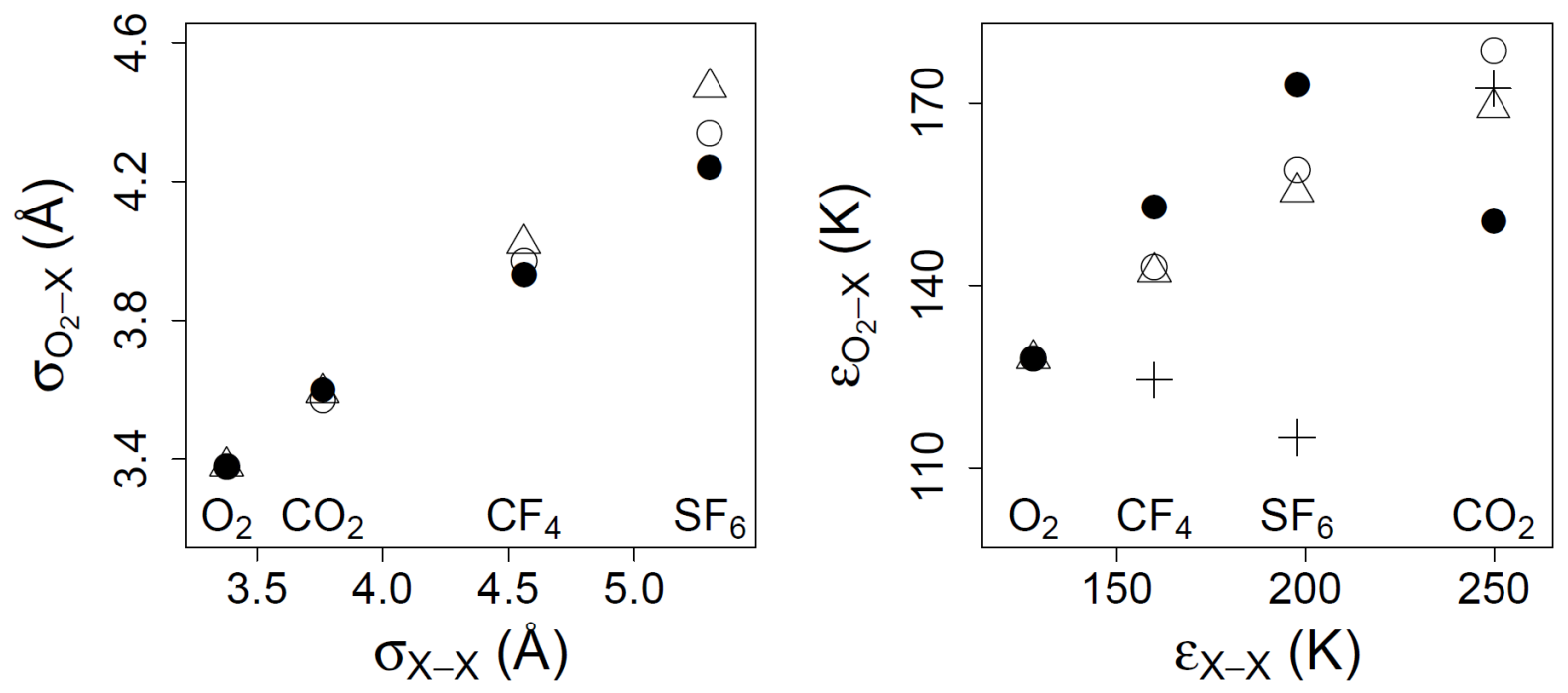

Figure 7. Combined parameters predicted with different combining rules for the interaction of $\mathrm{O}_{2}$ and three other species.

(a): collision diameters. 0 : fitted to experiment, $\bigcirc$ : Arithmetic mean, and $\triangle$ :Kong's rule.

(b): well depths. 0 : fitted to experiment $\bigcirc$ : Geometric mean, $\triangle$ : Harmonic mean, and + :

Kong's rule. 


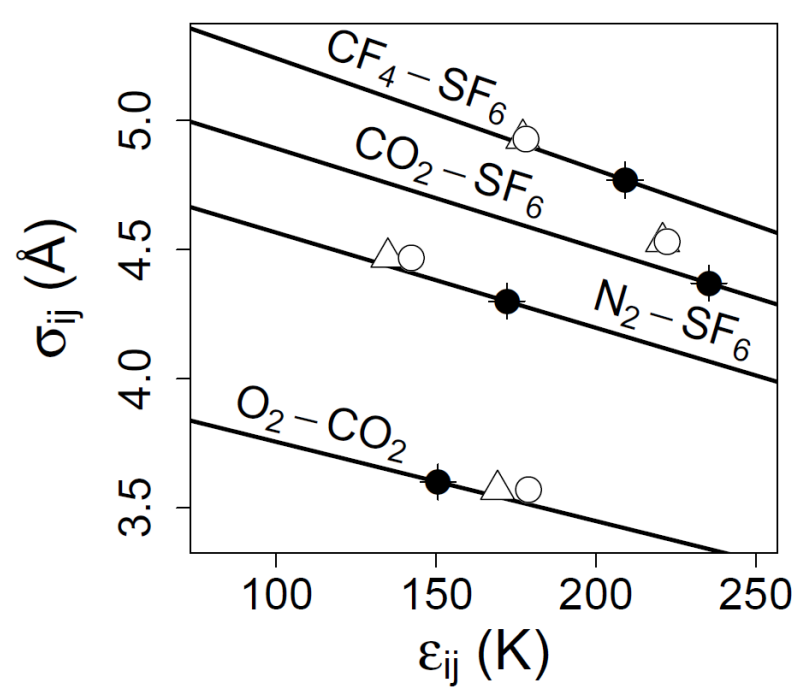

Figure 8 . Lines representing the trough for binary mixtures and combined parameters obtained by different methods.

: fitted to experiment, $\bigcirc$ : Geometric mean for $\varepsilon_{\mathrm{ij}}$ and arithmetic for $\sigma_{\mathrm{ij}}, \triangle$ : Harmonic mean for $\varepsilon_{\mathrm{ij}}$ and arithmetic for $\sigma_{\mathrm{ij}}$, and + : Kong's rule. Symbols are associated with the line they are closest to. 

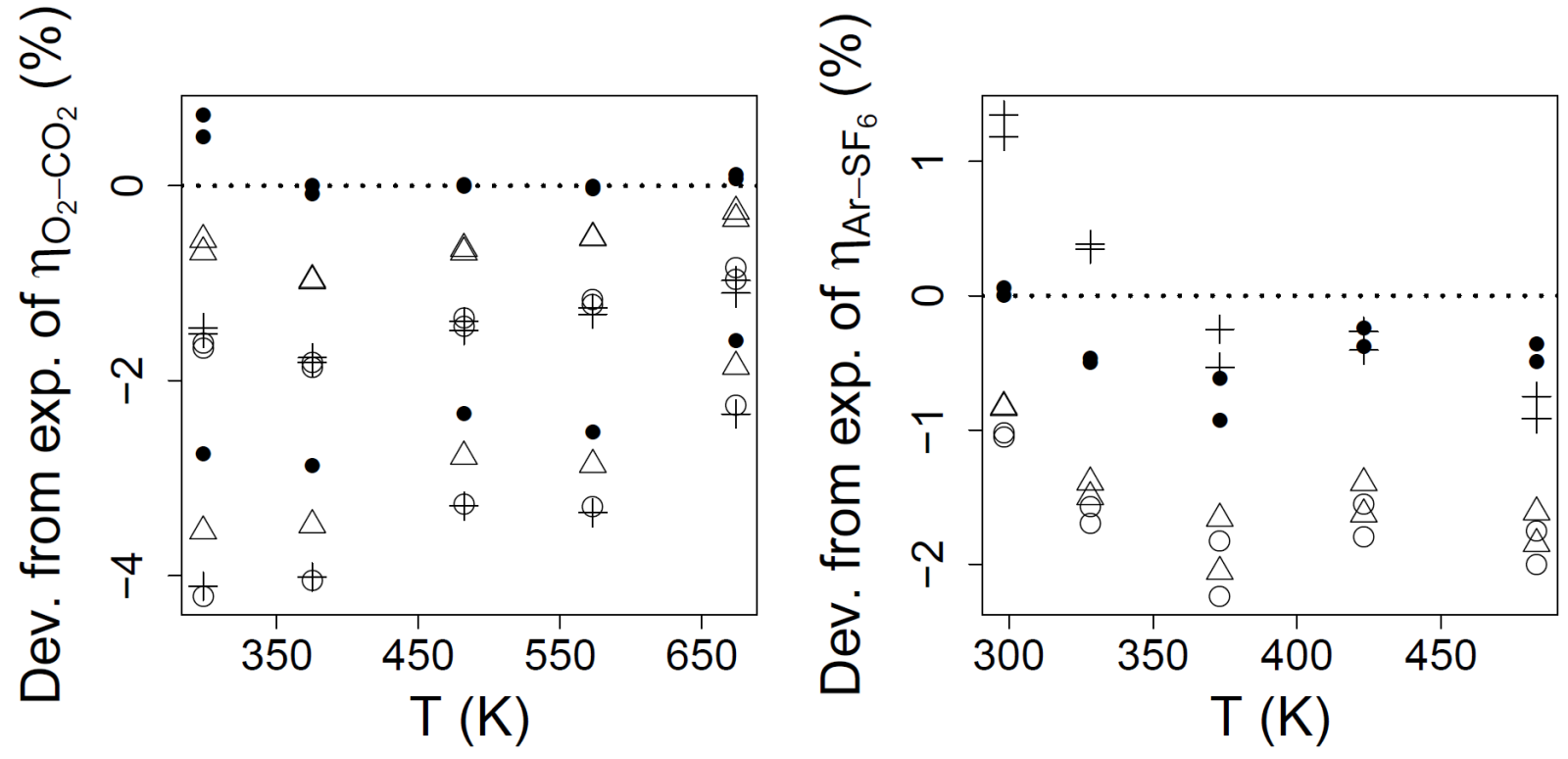

Figure 9. Deviation of the predicted viscosity relative to experimental values versus the temperature, using parameters obtained using different combining rules.

: fitted to experiment. $\bigcirc$ : Geometric mean for $\varepsilon_{\mathrm{ij}}$ and arithmetic for $\sigma_{\mathrm{ij}}, \triangle$ : Harmonic mean for $\varepsilon_{\mathrm{ij}}$ and arithmetic for $\sigma_{\mathrm{ij}}$, and + : Kong's rule. At each temperature, viscosity is measured for different ratios of the two species; hence the multiple points at each temperature. Measurements are from Kestin et al. (1997).[3] 


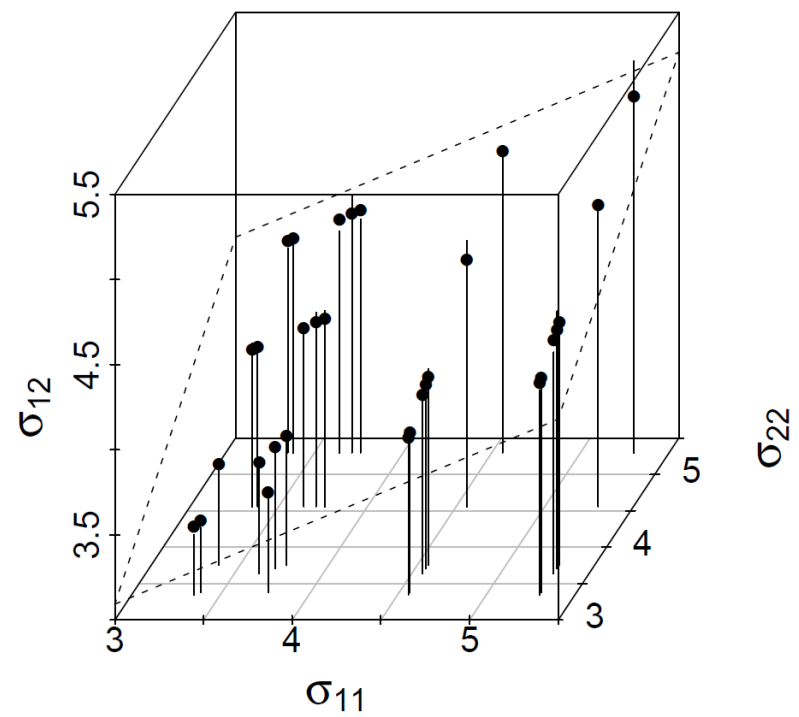

Figure 10. Collision diameters fitted by a plane.

Values are in $\AA$. The dashed lines represent the plane; The ends of the vertical lines represent the fitted collision diameters; 0 : Intersection with the plane. 